\title{
Numerical Analysis of Tightly Focused Spot for Confocal Microscopy Illumination by a Real Lens System
}

\author{
Rui Shi ${ }^{1,2^{*}}$, Site Zhang ${ }^{2}$, Christian Hellmann ${ }^{3}$ and Frank Wyrowski ${ }^{1}$ \\ 1. Applied Computational Optics Group, Friedrich Schiller University Jena, Jena, Germany \\ 2. LightTrans International UG, Jena, Germany \\ 3. Wyrowski Photonics UG, Jena, Germany \\ * Corresponding author: rui.shi@uni-jena.de
}

Confocal microscopy is widely used in both materials science and life sciences. It has good imaging performance due to the filtering of background noise. The illumination by a high numerical aperture condenser is essential for good image quality. It is studied quite intensively using the Debye-Wolf integral, which often assumes an aplanatic lens system [1]. However, the aberration of the misaligned system often affects the quality of the focal spot in experiments. The study of the focal spot based on the real condenser lens system is in high demand in order to analyze the experimental results, and furthermore, guide the experiment.

In this work, we perform the physical-optics modeling of the real condenser lens system with inclusion of full vectorial effects. The schematic of the illumination part of the confocal microscopy is shown in Fig. 1(a). The Gaussian wave source is generated in a fiber with a relative low divergence. It is collimated by a collimating lens (Edmund Optics: 49664) and then focused by a high numerical aperture condenser lens (Patent: US04384765; NA=0.95) to illuminate the sample.

The fully vectorial modeling is performed in the framework of field tracing [2,3] as shown in Fig. 1(b). We trace the vectorial field through different optical elements and free space by switching between different domains [4] with use of the Bi-directional (B) [5] and Propagation (P) operators [2,6], in order to maximize the accuracy and minimize the numerical effort.

The numerical experiment is performed in the software VirtualLab Fusion [7]. The focal spot is measured via energy density [1]. The wavelength of the illumination field is $632.8 \mathrm{~nm}$. The polarization is linear in the $y$ direction. The focal spots by the real lens system are shown in Fig. 2 (a) and (e) for circular and annular apertures respectively in perfect alignment. By comparing the results with those obtained by an ideal lens which is aplanatic via the Debye-Wolf integral [1], shown in Fig. 2 (b) and (f), we can conclude the real lens system is well designed and there are nearly no aberrations. For the situation in which the Gaussian source is misaligned with a lateral shift of $100 \mu \mathrm{m}$ in both the $x$ and $y$ directions, the simulation results are shown in Fig. 2 (c) and (g). We can see the focal spot deviates from the ideal one, especially in the case of the annular aperture. We compare the simulation results with those obtained by experiments from literature [8]; they are in good agreement. The misalignment of the lens system in the annular aperture case is more sensitive. The deviation in the focal spot is mainly caused by the misalignment of the lens system. More care should be taken when adjusting the system in the case of an annular aperture than in the case of a circular aperture. 
References:

[1] B. Richards and E. Wolf. Proceedings of the Royal Society of London A: Mathematical, Physical and Engineering Sciences 253(1274) (1959), p. 358.

[2] F Wyrowski and M Kuhn, Journal of Modern Optics 58 (2011), p. 449.

[3] R. Shi et al., SPIE Conference Proceedings (2018) 106940I

[4] F Wyrowski and C Hellmann, Proc. DGaO (2017).

[5] A v. Pfeil et al., Appl. Opt. 39 (2000), p. 3304.

[6] S Zhang et al., Appl. Opt. 55 (2016), p. 529.

[7] Fast physical optics software "Wyrowski VirtualLab Fusion", LightTrans GmbH, Jena, Germany.

[8] R Dorn, S Quabis, and G Leuchs. Journal of Modern Optics 50 (2003), p. 1917.

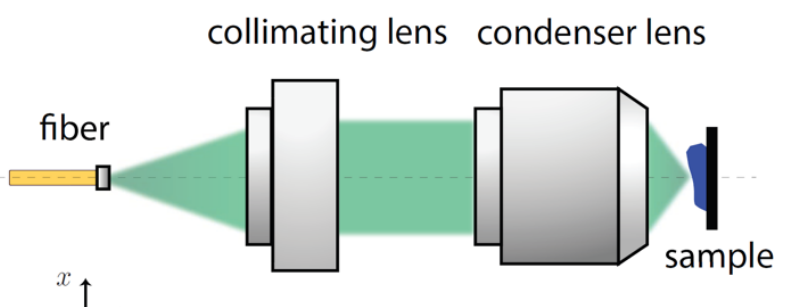

(a)

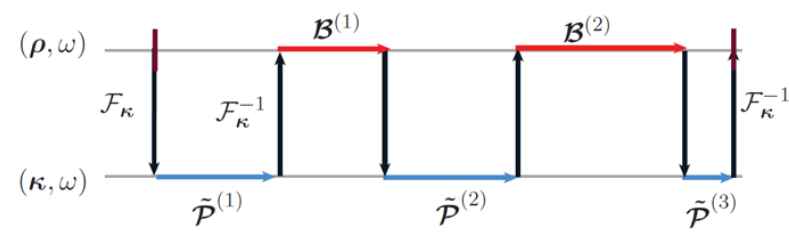

(b)

Figure 1. (a). The schematic of the illumination system for confocal microscopy. (b) The field tracing diagram for modeling the lens system and the propagation in the appropriate domains.
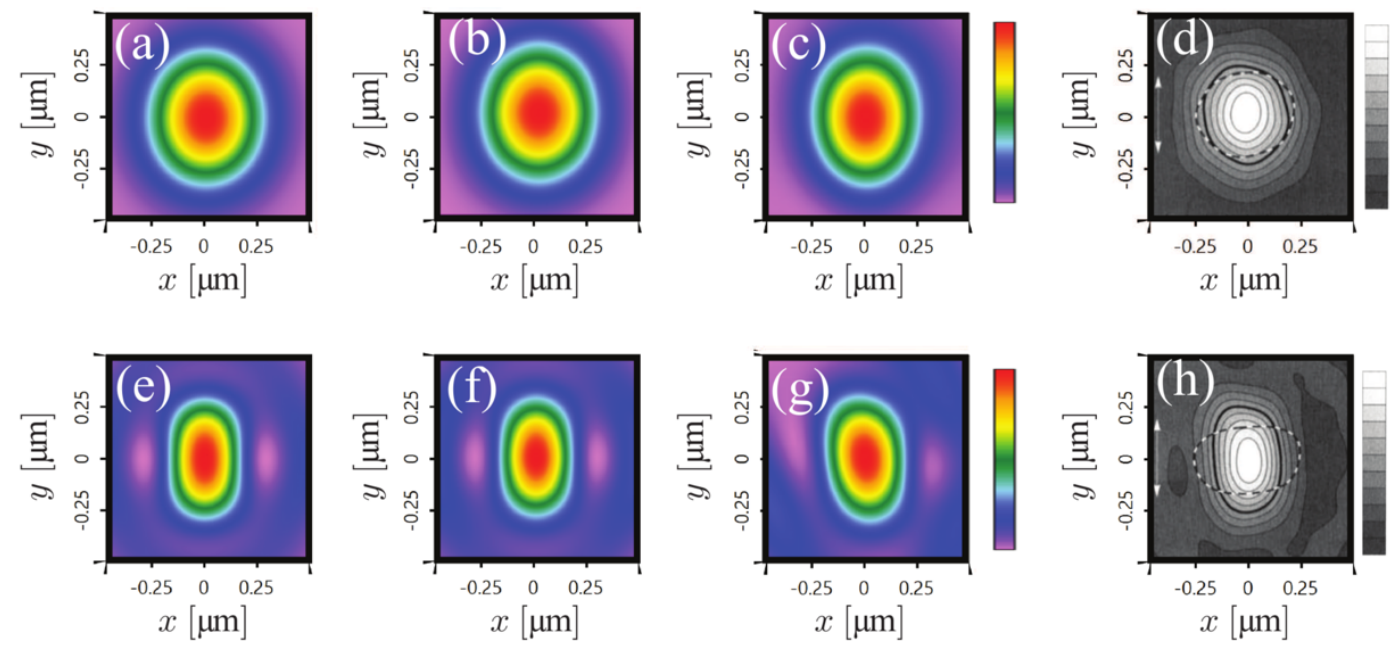

Figure 2. The top row corresponds to the case with a circular aperture. The bottom row is for the case of an annular aperture. (a) and (e) are the focal spots for the real lens system with perfect alignment. (b) and (f) are the focal spots with an ideal lens, which are obtained by the Debye-Wolf integral [1]. (c) and (g) are the focal spots when the source is misaligned with a shift of $100 \mu \mathrm{m}$ in both the $x$ and $y$ directions. (d) and (h) are the focal spots obtained experimentally, adapted from the literature [8]. 